\title{
Rab6-KIFL in a pinch
}

The targeting of intracellular membrane transport depends upon recognition events that are mediated through the Ras-related proteins, or Rabs. A search for partners of the Golgi-bound Rab6 protein using a two-hybrid screen identified the kinesin protein Rab6-KIFL. This kinesin was proposed to be involved in the transport of proteins within the Golgi and between the Golgi and ER, as well as in the partitioning of the Golgi during mitosis.

Recently, however, Hill et al. (EMBO J. 19, 5711-5719; 2000) have uncovered a new role for Rab6-KIFL in cytokinesis. Using immunofluorescence microscopy, they detected Rab6-KIFL in mitotic, but not interphase, cells. Although the protein appeared to be generally distributed in mitotic cells, the midbody of dividing HeLa cells was intensely stained. Levels of the protein were regulated in a similar way to cyclin B2, peaking during mitosis and rapidly falling thereafter.

These observations spurred a more detailed analysis of the function of this motor protein in cytokinesis. During metaphase, the protein is cytosolic, but as the spindle begins to elongate, it is present on the spindle, parallel to its axis. When the cell membrane begins to pinch during cytokinesis. Rab6-KIFL is found associated with short linear structures, which are most likely to be microtubules (the picture on the left shows anaphase II, that on the right shows telophase; Rab6 is shown in orange, DNA in blue and tubulin in green). Surprisingly, Hill and colleagues did not detect Rab6-KIFL in association with the Golgi at any point in the cell cycle. This finding calls into question previous contentions that this protein is involved in transport or Golgi distribution. Overexpression of fulllength Rab6-KIFL inhibited cell division and caused cell death, whereas transfection with the Rab6-binding domain alone did not affect division, although it was partially localized to the Golgi. Likewise, injection of
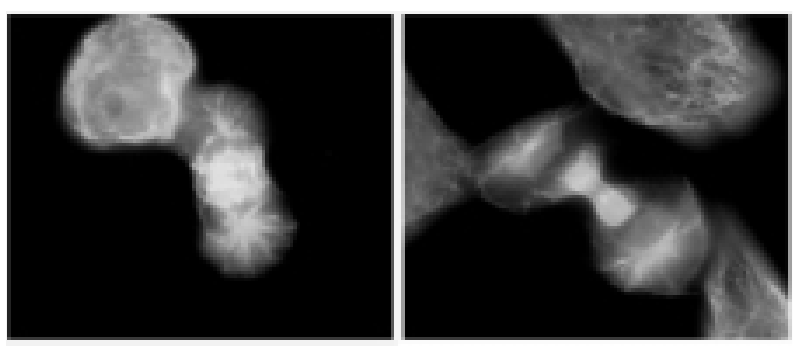

IMAGE COURTESY OF OXFORD UNIV. PRESS

cleavage furrow, but this then regressed to leave a binucleate cell. What of the two microtubule-binding domains? The MLKP1 family of kinesins is known to be required for cleavage-furrow formation. These kinesins also have two microtubule-binding domains and can crosslink microtubules in an antiparallel configuration. Studies using the Drosophila homologue, Zen-4, indicate that this motor protein, by stabilizing microtubules in such a manner, may help to mark the location at which the cleavage furrow will form. Another similarity is that MLKP1 has been found to bind to Arf1, a small GTPase that is involved in membrane transport. As we know that membrane-transport events are necessary during cytokinesis to pinch off the plasma membrane, this finding may explain the co-localization of Rab6-KIFL with Rab6 in the Golgi. These results indicate that perhaps the Rab6-KIFL motor protein is involved in the transport of Golgi fragments to the site of membrane fusion at the cleavage furrow.

ANGELA EGGLESTON anti-Rab6-KIFL antibody prevented cytokinesis - cells began to form a 